\title{
Indicações e propriedades mecânicas das resinas compostas convencionais e resinas compostas do tipo bulk-fill: revisão de literatura
}

\author{
Indications and mechanical properties of \\ conventional composite resins and bulk-fill \\ composites: literature review
}

\section{Letícia Meinberg Pedrosa' ${ }^{1}$ \\ Amanda de Oliveira Pinto Ribeiro ${ }^{2}$ (1) João Victor Frazão Câmara 3 (1) Josué Junior Araujo Pierote ${ }^{4}$ [C]}

\author{
'Universidade Federal do Rio de Janeiro (Rio de Janeiro). Rio de Janeiro, Brasil. leticia.m.pedrosa@gmail.com \\ ¿Universidade Estadual Paulista "Júlio de Mesquita filho" (São José dos Campos). São Paulo, Brasil. amandaribeiro11.2@gmail.com \\ 3Universidade de São Paulo (Bauru). São Paulo, Brasil. jvfrazao92@hotmail.com \\ ${ }^{4}$ Autor para correspondência. Universidade Estadual de Campinas (Piracicaba). São Paulo, Brasil. josuepierote@hotmail.com
}

\begin{abstract}
RESUMO | As resinas compostas são materiais restauradores amplamente utilizados nos consultórios odontológicos devido as suas propriedades mecânicas, estética e desempenho clínico. Contudo, a contração de polimerização presente nos compósitos convencionais ainda é um grande limitador. Neste contexto, foram desenvolvidas as resinas denominadas de bulk-fill, ou resinas de preenchimento, que possuem a possibilidade da inserção de um incremento único na cavidade dentária devido a uma menor contração de polimerização, melhores propriedades mecânicas, fácil execução, e como consequência a diminuição do tempo clínico. Todavia, ainda não há na literatura um consenso quanto à eficácia e durabilidade deste material. $\mathrm{O}$ objetivo deste estudo foi realizar uma revisão bibliográfica sobre as resinas do tipo bulk-fill e sua comparação com as resinas convencionais. Foram pesquisados artigos científicos em Português e em Inglês de 2002 a 2019 nas bases de dados Publicações Médicas (PUBMED) e Scientific Eletronic Library Online (SCIELO), sendo selecionados 50 trabalhos para leitura na íntegra a partir dos critérios de inclusão: idioma em Inglês ou Português e publicações realizadas nos últimos vinte anos e critérios de exclusão: artigos que não abordavam a temática central do estudo, publicações em outros idiomas que não fossem Inglês ou Português, artigos eletrônicos não disponíveis para a leitura completa e periódicos fora do período temporal estabelecido. De acordo com a bibliografia pesquisada, as resinas de preenchimento são uma opção de material restaurador, que vêm demostrando sucesso semelhante ao encontrado em reabilitações com as resinas compostas convencionais.
\end{abstract}

PALAVRAS-CHAVE: Polimerização. Dentística Operatória. Restauração Dentária Permanente.
ABSTRACT | Composite resins are restorative materials widely used in dental offices due to their mechanical properties, aesthetics, and clinical performance. However, the polymerization shrinkage present in conventional composites is still a major limitation. In this context, resins called bulk-fill, or filling resins, were developed, which can insert a single increment in the dental cavity due to less polymerization shrinkage, better mechanical properties, easy execution, and the consequent decrease in clinical time. However, there is still no consensus in the literature regarding the effectiveness and durability of this material. The objective of this study was to carry out a bibliographic review on bulk-fill resins and their comparison with conventional resins. Scientific articles in Portuguese and English from 2002 to 2019 were searched in the databases Medical Publications (PUBMED) and Scientific Electronic Library Online (SCIELO), with 50 papers selected for full reading based on the inclusion criteria: language in English or Portuguese and publications made in the last twenty years and exclusion criteria: articles that did not address the central theme of the study, publications in languages other than English or Portuguese, electronic articles not available for full reading and periodicals outside the established period. According to the researched bibliography, filling resins are an option of restorative material, which has been showing success similar to that found in rehabilitation with conventional composite resins.

KEYWORDS: Polimerization. Dentistry Operative. Dental Restoration Permanent. 


\section{Introdução}

A busca por materiais restauradores que possuem características semelhantes as unidades dentárias têm acarretado ao grande desenvolvimento das resinas compostas, quanto aos aspectos físicos, mecânicos e estéticos. ${ }^{1}$ Atualmente, estão disponíveis no mercado diversos tipos de resinas compostas. Uma nova classe de compósitos diretos está ganhando espaço nos consultórios odontológicos, denominadas de bulk-fill.?

A principal característica a ser melhorada nos compósitos diretos, segundo Caneppele e Bresciani ${ }^{3}$, é a contração de polimerização, que pode acarretar o rompimento da ligação entre a interface dente/material restaurador. ${ }^{4,5}$ De acordo com Silva et al. ${ }^{4}$, após a polimerização da resina composta pode haver perda de volume do material em até 3\%, podendo ocasionar alterações maléficas, diminuindo a vida útil da restauração.

A técnica de inserção incremental é recomendada quando se utiliza as resinas compostas convencionais, visando uma diminuição da contração de polimerização. A adaptação de pequenos volumes de incrementos de resina na cavidade ocasiona uma menor tensão marginal, quando comparada a técnica de inserção de incremento único. Contudo, estudos afirmam que nenhum método de inserção incremental pôde impedir a microinfiltração marginal. ${ }^{6}$

Neste contexto, muitas pesquisas vêm desenvolvendo materiais restauradores que possuem melhores características quanto a contração de polimerização. As resinas compostas do tipo bulk-fill, ou de preenchimento, possuem menor contração de polimerização ${ }^{7}$, e por permitirem a inserção na cavidade de incrementos de até $4 \mathrm{~mm}$, há a possibilidade da diminuição de erros do operador e de incorporação de bolhas, além de uma melhor ligação na interface dente/restauração. ${ }^{3}$

Este estudo objetivou a realização de uma pesquisa na literatura sobre as resinas compostas do tipo bulk-fill e sua comparação com os compósitos convencionais. Foi realizada um breve histórico das resinas compostas, suas características, tipos, indicações e técnicas de execução.

\section{Materiais e Métodos}

Uma pesquisa de caráter bibliográfico foi realizada nas bases de dados Publicações Médicas (PUBMED) e Scientific Eletronic Library Online (SCIELO) por meio de artigos científicos eletrônicos que abordavam o seguinte tema: Resinas Compostas Convencionais $x$ Resinas Compostas do tipo bulk-fill: revisão de literatura.

Foram utilizados os Descritores em Ciências da Saúde (DeCS), cadastrados no site da Biblioteca Virtual em Saúde (BVS): Polimerização, Dentística Operatória, Restauração Dentária Permanente. Os descritores foram buscados sozinhos e combinados. Foram selecionados artigos publicados no período de 2002 a 2019, além de livros e artigos referências no tema publicados anteriormente.

Os critérios de inclusão utilizados durante o processo de filtragem foram: idioma em Inglês ou Português e publicações realizadas nos últimos vinte anos. Os critérios de exclusão foram: artigos que não abordavam a temática central do estudo, publicações em outros idiomas que não fossem Inglês ou Português, artigos eletrônicos não disponíveis para a leitura completa e periódicos fora do período temporal estabelecido para esta revisão.

Primeiramente, foram selecionados artigos nas bases de dados de acordo com a pesquisa dos descritores. Em seguida, foi realizada a leitura dos títulos dos artigos encontrados nas bases de dados. Posteriormente, uma pré-seleção foi feita para a análise do resumo/ abstract. Foram selecionados 50 artigos científicos e 4 livros complementares para leitura na íntegra e inclusão no presente estudo.

\section{Revisão de Literatura}

\section{Histórico}

As resinas compostas foram introduzidas como material restaurador odontológico no fim dos anos 50 e início da década de 60, por Bowen. 0 início do desenvolvimento deste material se deu ao reforçar resinas epóxicas e ácido metacrílico (dimetacrilatos) com grande partículas de carga, como pó de quartzo. ${ }^{8}$ 
A incorporação de partículas de carga à matriz de resina aumentou significativamente suas propriedades. Inicialmente, utilizava-se quartzo por ser duro e ter um índice de refração de luz semelhante ao esmalte dentário, porém a dureza do quartzo tornava difícil o polimento dessas resinas pois, por serem grandes, as partículas se perdiam facilmente tornando a superfície da restauração muito áspera e sujeita a manchas. Em uma tentativa de minimizar estes problemas os fabricantes reduziram gradualmente o tamanho das partículas. $\stackrel{9}{ }$

Com a diminuição do tamanho das partículas foi possível aumentar o percentual de carga inorgânica das resinas compostas e, consequentemente houve a redução da contração de polimerização e melhorou a distribuição de carga. ${ }^{10}$ Isto foi possível devido ao advento da nanotecnologia que, reduzindo o tamanho das partículas de carga possibilitou a redução do estresse de polimerização e o aumento da resistência ao desgaste..$^{11}$

A redução no tamanho das partículas desencadeou a produção dos compósitos denominados resinas microparticuladas, constituídas de partículas de sílica coloidal com tamanho variando entre 0,02 a 0,04 $\mu \mathrm{m}$. As resinas microparticuladas, apesar de apresentarem excelente polimento, possuem alto índice de contração de polimerização devido à pouca porcentagem de carga. ${ }^{12}$ Para aumentar a quantidade dessas partículas se utilizou uma nova carga com resina composta pré-polimerizada altamente carregada com partículas de sílica coloidal que foram então misturadas à matriz de resina, chegando assim, a cerca de $80 \%$ em peso ou $70 \%$ em volume.

O próximo passo de desenvolvimento das resinas compostas foi alcançado combinando partículas de tamanho convencional com micropartículas. Estes materiais são os compósitos híbridos e possuem mais de $80 \%$ em peso ( 60 a $70 \%$ de volume) de partículas de carga e podem ser utilizados para a restauração de dentes anteriores e posteriores, apresentando indicação "universal". 13

As modificações mais atuais se atentam a mudanças na matriz polimérica do material com o objetivo de desenvolver sistemas com menor contração de polimerização. ${ }^{14}$

\section{Contração de polimerização}

A polimerização é uma das etapas de maior importância nos procedimentos de reabilitações com resinas compostas e a contração de polimerização é uma consequência inerente a técnica. $O$ estresse decorrente desta contração pode ocasionar em alterações que comprometam a qualidade e durabilidade da restauração. ${ }^{4}$ fotopolimerização é um dos motivos que ocasionam falhas clínicas precoces nas restaurações devido a negligência ou falta de conhecimento do profissional.?

A polimerização dos compósitos é o processo da modificação de monômeros em cadeias de polímeros. A formação de cadeias lineares ou ramificadas dependerá das propriedades e quantidade de luz emitida pelo fotoativador. Neste processo, devido à redução volumétrica do compósito, há como intercorrência a formação de uma tensão na interface. ${ }^{15}$ Diversos fatores contribuem para a contração de polimerização, como a extensão e o fator de configuração (Fator C) da cavidade, qualidade e composição do compósito, técnica utilizada, modo de fotoativação e a habilidade do profissional operador. $\frac{16}{6}$

Visando a diminuição da contração de polimerização de resinas compostas convencionais, é necessário que se utilize a técnica incremental. ${ }^{17}$ As resinas bulk-fill foram desenvolvidas no contexto de melhorar esta questão. Estas resinas possuem baixo grau de polimerização, possibilitando a inserção de incremento único. ${ }^{3}$

Estes compósitos apresentam composições de matriz orgânica diferentes das encontradas nas resinas compostas convencionais, e podem também apresentar diferentes fotoiniciadores. Para o sucesso nas restaurações com este tipo de resinas é necessário a utilização de aparelhos fotoativadores à base de LED (diodo emissor de luz azul) com alta potência, visando a correta conversão de polímeros formados.?

A polimerização de resinas compostas através de LED azul surgiu no intuito de superar as desvantagens da fotoativação através da luz halógena. Por meio dos feixes de semi-condutores destes fotativadores, é possível diminuir o aquecimento, além do seu comprimento de onda ser mais específico para a ativação da canforoquinona quando comparado com a luz halógena. ${ }^{18}$ 
A longevidade e o sucesso das restaurações com compósitos estão diretamente relacionados com a fotopolimerização. Com isso, para que os fotopolimerizadores LED desempenhem suas funções com êxito, é necessário que possuam as características ideais, entre elas o tamanho de onda, potência adequada e tipo de pulso $\frac{19}{19}$, visando a correta conversão de monômeros em polímeros. $\underline{\underline{20}}$

\section{Indicações}

De acordo com Ferracane ${ }^{14}$, há uma ampla variedade de compósitos com diferentes características de manuseio, propriedades mecânicas e estéticas. O sucesso das restaurações com resinas compostas e durabilidade é decorrente da condição do substrato dental a ser restaurado, do sistema adesivo e protocolo de aplicação, da acurácia do profissional operador, além das condições bucais as quais as resinas são submetidas. 21

As resinas bulk fill oferecem translucidez maior do que as encontradas nos compósitos convencionais. Esta característica facilita uma maior penetração dos feixes de luz emitidas pelo fotopolimerizador até as camadas mais profundas da restauração e nos ângulos de maior dificuldade de acesso ${ }^{22}$, e segundo Taubock et al. $\underline{23}$, este efeito está relacionado com um maior grau de conversão, e consequentemente a meIhoria nas propriedades físicas.

A alta translucidez encontrada nestes compósitos influencia de forma negativa na aparência estética das restaurações, deixando-as acinzentadas, sendo um limitador para áreas que apareçam mais no sorriso. Dessa forma, as resinas bulk-fill são indicadas para a restauração em dentes posteriores, devido a melhoria de propriedades físicas e biológicas, apresentando bom desempenho ao longo prazo. ${ }^{1}$

De acordo com Ehlers et al., resinas do tipo bulk-fill podem ser utilizadas com êxito em restaurações de dentes decíduos. ${ }^{24}$ Mosharrafian et al. afirmaram que estes compósitos são indicados para classe II de unidades posteriores decíduas, apresentando propriedades semelhantes às encontradas nos compósitos convencionais em relação a microinfiltração, visando a diminuição do tempo do tratamento pediátrico. ${ }^{25}$
Estas resinas também podem ser utilizadas em dentes anteriores decíduos severamente danificados. ${ }^{26}$

\section{Características}

Os compósitos dentários podem ser classificados de acordo com algumas diferenças em sua formulação e consequente indicação clínica. O sistema de resina BisGMA/TEGDMA (ou similar) tem uma capacidade limitada para reduzir estresse de contração, sem reduções subsequentes de conversão e das propriedades dos polímeros. ${ }^{27}$ As resinas bulk-fill possuem contração volumétrica possivelmente reduzida, entretanto a composição é muito variável, sendo que cada fabricante apresenta sua própria tecnologia. Dentre os princípios utilizados pelos diversos fabricantes, pode-se citar a utilização de monômeros específicos, monômeros coadjuvantes, diferentes fotoiniciadores, inclusão de diferentes cargas inorgânicas (fibras de vidro) e utilização de energia ultrassônica anteriormente à fotopolimerização. $\underline{3}$

As resinas de preenchimento podem ser encontradas de duas formas: Resinas "bulk-fill tipo pasta" e "bulk-fill tipo flow"28, diferindo sua técnica de acordo com a apresentação. É necessário a identificação das características de cada material de acordo com o fabricante. ${ }^{3}$

O uso dos compósitos do tipo "bulk-fill flow" podem ser utilizadas como forramento ou para restaurações diretas. ${ }^{29} \mathrm{O}$ preenchimento de paredes pulpares em cavidades profundas é vantajoso, visto que seu escoamento permite o preenchimento de áreas estreitas e com ângulos de difícil acesso. Já as resinas "bulkfill" do tipo pasta são indicadas para restaurações diretas em cavidades de até $4 \mathrm{~mm} . \underline{5}$

Estes compósitos também podem ser classificados quanto a cura, podendo ser dual (polimerização química e através de fotoativação) ou apenas através de fotoativação. Outra especificação é quanto à necessidade de uma cobertura com uma resina convencional. Todas as resinas bulk-fill podem ou não serem cobertas por resinas convencionais, contudo, há fabricantes que caracterizam esse recobrimento como imprescindível. Alguns exemplos dessas resinas são: Dentsply - SDR, 3M ESPE - Filtek Bulk-Fill Flowable, Heraeus Kulzer - Venus Bulk-Fill, Ivoclar Vivadent Tetric EvoFlow Bulk-Fill, e Voco - x-tra base..$^{30}$ 


\section{Técnica incremental x Técnica preenchimento único}

A técnica restauradora mais indicada na utilização das resinas compostas convencionais é a técnica incremental. A redução da tensão de contração é influenciada pela quantidade do volume de material, a configuração da cavidade e o contato mínimo com paredes opostas durante a polimerização. Com a utilização desta técnica, a tensão de contração é menor devido ao volume reduzido de material. $\underline{31}$

A técnica incremental consiste nas múltiplas inserções de incrementos menores de $2 \mathrm{~mm}$ de resina composta na cavidade em forma de cunha. Cada incremento é fotopolimerizado individualmente. Esta técnica reduz o fator $\mathrm{C}$ e evita a distorção das paredes da cavidade. Cada incremento inserido vai ser compensado pelo próximo a ser colocado e, com isso, a contração de polimerização é menor. Apenas a última camada inserida pode danificar a superfície de ligação com sua redução de volume. $\underline{\underline{32}}$

Com o desenvolvimento da resina do tipo bulk-fill surgiu a tecnologia de preenchimento em massa que permite a inserção do compósito em um incremento único de 4-5mm de profundidade, sem que haja comprometimento da polimerização. ${ }^{15,33} \mathrm{~A}$ exposição à luz de 20 segundos foi o suficiente para polimerização da resina bulk-fill nesse volume $(4 \mathrm{~mm}) .22$ Entretanto, de acordo com Veiga e Vasconcellos ${ }^{34}$, quando se utiliza resina bulk-fill tipo pasta em cavidades profundas em único incremento de até $4 \mathrm{~mm}$ com tempo de fotopolimerização semelhante a técnica incremental, há um menor grau de conversão da resina da base da restauração, pois o total da quantidade de vezes que o incremento é fotopolimerizado vai ser menor, o que pode influenciar na dureza do material.

Já segundo Obeid et al. $\underline{20}$ a eficácia da fotoativação em incrementos de $2 \mathrm{~mm}$ para $4 \mathrm{~mm}$ não houve variação significativa. $O$ processo de polimerização está intimamente ligado a conversão de monômeros em polímeros de resina composta e essa conversão está relacionada com a composição da resina..$\underline{20}$

As duas categorias de compósitos bulk-fill (fluido e tipo pasta) possuem propriedades físicas diferentes, como a tensão de polimerização. $\frac{33}{} \mathrm{~A}$ resina bulk-fill do tipo flow pode ser usada com ponta de seringa, proporcionando melhor acesso, menor contração volumétrica e menos estresse na interface durante a polimerização. ${ }^{35}$ Esse material fluido requer preenchimento oclusal com resina composta convencional, já a bulk-fill em pasta não necessita do preenchimento oclusal com outro tipo de resina. ${ }^{36}$ Essas apresentam cargas mais altas e consequentemente são mais resistentes ao desgaste. ${ }^{37}$

Os compósitos diretos do tipo bulk-fill foram desenvolvidos no intuito da diminuição de erros dos profissionais operantes, devido a técnica de incremento, diminuindo o tempo de trabalho, e as chances de microbolhas, e consequentemente uma melhor interface adesiva entre o dente e restauração. Quando comparados com as resinas convencionais, as resinas de preenchimento desempenham melhor adaptação na cavidade, aspectos físicos elevados e boa aptidão de manipulação. $\underline{38}$

Em relação a estética, a técnica de incremento único impossibilita a estratificação, muitas vezes necessária para reabilitações estéticas em dentes anteriores. ${ }^{39}$

\section{Propriedade mecânica}

O sucesso das restaurações em resina composta está associado às suas propriedades mecânicas, sendo sua composição um fator predominante na influência do comportamento. $\frac{27}{} \mathrm{Com}$ o aumento do teor de carga, a contração de polimerização, o coeficiente de expansão linear e a absorção de água são reduzidas, em contrapartida aumentam-se a resistência à compressão, desgaste à tração e módulo de elasticidade. 40

As resinas bulk-fill do tipo pasta possuem composição química muito semelhante aos compósitos de

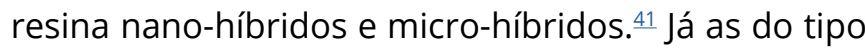
flow normalmente apresentam menor volume de carga, menor módulo de elasticidade e menor dureza quando comparada às resinas convencionais ou as resinas bulk-fill na forma de pasta. $\underline{42}$

Os compósitos de preenchimento apresentam como característica, segundo Christensen ${ }^{43}$ uma alta resistência à flexão, podendo suportar cargas de compressão e desgaste, mantendo a forma anatômica preconizada pelo cirurgião-dentista durante a execução da restauração. 


\section{Discussão}

A contração de polimerização é um dos principais aspectos que necessita atenção quando se utiliza resina composta como material restaurador. Diferentes fatores contribuem para este estresse, como a extensão e o fato de configuração (Fator C) da cavidade ${ }^{16}$, como também o tipo de carga e distribuição, monômeros da resina e técnica de fotoativação. O sucesso clínico das restaurações com resinas compostas está relacionado diretamente com os aparelhos de LED utilizados na fotopolimerização. Entender os mecanismos e conceitos do fotopolimerizador otimiza o resultado clínico. $\underline{24}$

Os compósitos de preenchimento apresentam menor retração e tensão de polimerização. 44 Souza et al. $\frac{16}{}$ afirmaram que a composição é o fator que mais influencia no estresse de contração. $O$ estudo publicado por Freitas et al. ${ }^{45}$ objetivou comparar a força de contração de polimerização de dois compósitos diferentes, um convencional e outro do tipo bulk-fill. A resina convencional polimerizada através da técnica de fotopolimerização convencional apresentou menor força de contração como resultado.

Na pesquisa in vitro publicada por Charamba et al. $\frac{5}{\text {, }}$ o compósito convencional apresentou menor resistência de união quando comparado aos compósitos do tipo bulk-fill. Em relação à microinfiltração, segundo Mosharrafian et al. ${ }^{25}$, resinas convencionais e de preenchimento não são significantemente diferentes.

De acordo com Costa et al. .46 , os autores obtiveram resultados clínicos considerados esteticamente satisfatórios nas restaurações com resinas bulk-fill. Contudo, a alta translucidez encontrada nestes compósitos pode desencadear restaurações com alto valor, com cores acinzentadas.

Barutcigil et al. ${ }^{47}$ afirmaram que após a imersão em bebidas e a longo prazo, as cores das resinas compostas bulk-fill pesquisadas mudaram significativamente. Já no trabalho de Almeida et al. ${ }^{48}$, dentre os compósitos analisados, a resina bulk-fill avaliada apresentou menor manchamento após a exposição ao café, independentemente do sistema de polimento utilizado. Miletic et al. ${ }^{49}$ afirmaram que se pode esperar alterações de cor semelhantes nos compósitos de preenchimento e nos convencionais.
Em relação ao desempenho clínico, a revisão sistemática publicada por Veloso et al.50 concluiu que em um período de 12 a 72 meses de acompanhamento, as resinas de preenchimento e as convencionais foram semelhantes estatisticamente, sendo as resinas bulk-fill uma opção de tratamento para restaurações diretas em dentes posteriores. 44

Em um estudo mecânico in vitro realizado por Abdulmajeed et al. ${ }^{51}$, foram comparadas uma resina bulk-fill com uma resina convencional. Foi demonstrado mínima diferença nas propriedades mecânicas de ambas as resinas. Corroborando com os achados de Sunbul et al. $\frac{52}{}$, em que o compósito bulk-fill em pasta testado mostrou resultados comparáveis a outros compósitos microhíbridos convencionais.

O estudo in vitro conduzido por Rauber et al. $\frac{53}{\text { re- }}$ portou, através dos resultados do comportamento de fadiga, que os dentes com cavidades MOD classe II restaurados com compósito bulk-fill do tipo pasta resultou em resistência à fadiga semelhantes às cavidades restauradas com compósito nanohíbrido na técnica incremental.

Já no estudo in vitro realizado por Rosatto et al. ${ }^{41}$ que investigou a resistência à fratura de dentes com restaurações MOD classe II, foram encontrados valores significativamente mais baixos para molares restaurados com resina convencional na técnica incremental do que com compósitos bulk-fill na técnica de preenchimento único. Além disso, técnicas de preenchimento com bulk-fill resultaram em menor deflexão de cúspide e maior resistência à fratura.

Papadogiannis ${ }^{54}$ confirmou que o uso de camada de compósito convencional como cobertura de restaurações com bulk-fill de base fluida é obrigatório para alcançar maior resistência, o que pode ser explicado pelos achados de Rosatto et al.41, que demonstrou um comportamento mecânico menos eficaz das resinas bulk-fill do tipo flow do que bulk-fill tipo pasta e compósitos convencionais.

Em relação à técnica de inserção a ser utilizada, o estudo realizado por Van Ende et al. ${ }^{22}$ avaliou três tipos de resinas: um compósito universal fluido, uma resina bulk-fill flow e uma resina convencional, em diferentes configurações de cavidade. Foi demonstrado que a técnica de preenchimento e o tipo de resina 
pode ter um grande impacto na adesão do compósito, principalmente em cavidades com alto fator $C$. A resina bulk-fill flow forneceu boa resistência de união independente da técnica de preenchimento e profundidade da cavidade, entretanto quando os compósitos convencionais foram utilizados na técnica de preenchimento único a adesão não foi satisfatória.

Outro estudo avaliou a qualidade das margens proximais de restaurações realizadas com resina bulkfill com um único incremento e restaurações com resina convencional em três incrementos. Não foram observadas diferenças significativas entre os dois tipos de técnicas restauradoras e suas respectivas resinas. ${ }^{36}$ Dessa forma, é importante que a técnica esteja de acordo com o tipo da resina escolhida para a restauração.

A revisão de literatura realizada por Van Ende et al. ${ }^{37}$ sugeriu a melhor indicação de resinas bulk-fill flow para cavidades estreitas (menores que $4 \mathrm{~mm}$ ) e principalmente em cavidades de difícil acesso. Já em cavidades mais extensas, as resinas bulk-fill em pasta com maior quantidade de carga são preferíveis.

\section{Considerações Finais}

De acordo com a revisão bibliográfica realizada, conclui-se que as resinas bulk fill são eficazes. Podem ser indicadas para restaurações em dentes posteriores e dentes decíduos. Possuem melhores propriedades mecânicas, menor contração de polimerização e técnica de fácil execução quando comparada as resinas convencionais, o que ocasiona a diminuição de erros e um menor tempo clínico. Se fazem necessários mais estudos in vivo para melhor elucidação do desempenho clínico das restaurações com resinas de preenchimento a longo prazo.

\section{Contribuições dos autores}

Pedrosa LM escreveu e corrigiu o artigo. Ribeiro AOP e Câmara JVF escreveram o artigo. Pierote JJA idealizou e supervisionou a execução do artigo.

\section{Conflitos de interesses}

Nenhum conflito financeiro, legal ou político envolvendo terceiros (governo, empresas e fundações privadas, etc.) foi declarado para nenhum aspecto do trabalho submetido (incluindo, mas não se limitando a subvenções e financiamentos, participação em conselho consultivo, desenho de estudo, preparação de manuscrito, análise estatística, etc.).

\section{Referências}

1. Ferreira GC, Mizael VP, Guimarães BM, Araújo TGF. Nova geração de resinas compostas bulk-fill. Arq bras odontol [Internet]. 2019;14(2):1-6. Disponível em: http://periodicos. pucminas.br/index.php/Arquivobrasileirodontologia/article/ view/21418

2. Amaral RC, Ilkiu RE, Sinhoreti MAC. Eficácia da polimerização das resinas compostas Bulk-fill com as atuais fontes à base de LEDs Eficácia da polimerização das resinas compostas Bulk-fill com as atuais fontes à base de LEDs. Dicas [Internet]. 2015;4(4). Disponível em: https://www.academia.edu/37472060/ Efic\%C3\%A1 cia_da_polimeriza\%C3\%A7\%C3\%A30_das_resinas_ compostas_Bulk_fill_com_as_atuais_fontes_\%C3\%A0_base_de_ LEDS

3. Caneppele TMF, Bresciani E. Resinas bulk-fill - O estado da arte. Rev Assoc Paul Cir Dent [Internet]. 2016;70(3):242-8. Disponível em: http://revodonto.bvsalud.org/scielo.php?pid=S000452762016000300003\&script=sci_arttext\&tIng=pt

4. Silva FJV, Silva EL, Januário MVS, Vasconcelos MG, Vasconcelos RG. Técnicas para reduzir os efeitos da contração de polimerização das resinas compostas fotoativasas. Salusvita [Internet]. 2017;36(1):187-203. Disponível em: https://secure. unisagrado.edu.br/static/biblioteca/salusvita/salusvita_v36 n1 2017 art 13.pdf

5. Charamba CF, Meireles SS, Duarte RM, Montenegro RV, Andrade AKM de. Resistência de união de compósitos do tipo Bulk Fill: análise in vitro. Rev Odontol da UNESP. 2017;46(2):77-81. https://doi.org/10.1590/1807-2577.15216

6. Ribeiro MA, Ferreira IX, Lima RP, Mariz ALA, Pompeu JGF, Silva CHV. Influência da Técnica de Inserção de Resina Composta sobre o selamento marginal em restaurações estéticas oclusais. Odontol. Clín.-Cient [Internet]. 2010;9(4):345-8. Disponível em: http://revodonto.bvsalud.org/scielo.php?script=sci_ arttext\&pid=S1677-38882010000400014

7. Alves KC, Lucena RP, Souza FB. Esthetic Reanatomization in Posterior Teeth with Bulk Fill Resins: Case Report. J Heal Sci. 2019;21(5):470-3. https://doi.org/10.17921/24478938.2019v21n5p470-473 
8. Bowen RL. Properties of a silica-reinforced polymer for dental restorations. J Am Dent Assoc. 1963;66(1):57-64. http://dx.doi. org/10.14219/jada.archive.1963.0010

9. Phillips RW. Materiais dentários de Skinner. $9^{a}$ ed. Rio de Janeiro: Guanabara; 1993.

10. Mondelli J. Restaurações Estéticas. São Paulo: Sarvier; 1984.

11. Cramer NB, Stansbury JW, Bowman CN. Recent advances and developments in composite dental restorative materials. J Dent Res. 2011;90(4):402-16. https://dx.doi. org/10.1177\%2F0022034510381263

12. Anusavice KJ. Materiais Dentários. $10^{a}$ ed. São Paulo: Guanabara Koogan; 2002.

13. Baratieri LN. Dentística - Procedimentos preventivos e restauradores. São Paulo: Santos; 1992.

14. Ferracane JL. Resin composite - State of the art. Dent Mater. 2011;27(1):29-38. https://doi.org/10.1016/j.dental.2010.10.020

15. Ilie N, Keßler A, Durner J. Influence of various irradiation processes on the mechanical properties and polymerisation kinetics of bulk-fill resin based composites. J Dent. 2013;41(8):695702. https://doi.org/10.1016/j.jdent.2013.05.008

16. Pires-de-Souza FCP, Drubi Filho B, Casemiro LA, Garcia LFR, Consani S. Polymerization shrinkage stress of composites photoactivated by different light sources. Braz Dent J. 2009;20(4):319-24. https://doi.org/10.1590/S0103$\underline{64402009000400010}$

17. Sakaguchi RL, Douglas WH, Peters MCRB. Curing light performance and polymerization of composite restorative materials. J Dent. 1992;20(3):183-8. https://doi.org/10.1016/03005712(92)90136-Z

18. Caughman WF, Rueggeberg FA, Curtis Jr JW. Clinical guidelines for photocuring restorative resins. J Am Dent Assoc. 1995;126(9):1280-2. https://doi.org/10.14219/jada. archive.1995.0364

19. Silva Neto JMA, Santana LR, Lima KFF, Medeiros MLBB, Cavalcanti TC. O uso das resinas compostas tipo bulk fill: Uma revisão de literatura. Rev Eletrônica Acervo Saúde. 2019;(37):e1887. https://doi.org/10.25248/reas.e1887.2019

20. Obeid AT, Coneglian LC, Albergaria LS, Scotti CK, Bombonatti JFS. Efeito de diferentes protocolos fotoativadores e espessuras dos incrementos na dureza de resinas Bulk fill. Rev Odontol Arac [Internet]. 2020;41(2):34-9. Disponível em: https://pesquisa. bvsalud.org/portal/resource/pt/biblio-1102682

21. Schwendicke F, Göstemeyer G, Blunck U, Paris S, Hsu LY, Tu YK. Directly placed restorative materials: Review and network meta-analysis. J Dent Res. 2016;95(6):613-22. https://doi. org/10.1177/0022034516631285
22. Van Ende A, De Munck J, Van Landuyt KL, Poitevin A, Peumans $M$, Van Meerbeek B. Bulk-filling of high C-factor posterior cavities: Effect on adhesion to cavity-bottom dentin. Dent Mater. 2013;29(3):269-77. http://dx.doi.org/10.1016/j.dental.2012.11.002

23. Tauböck TT, Jäger F, Attin T. Polymerization shrinkage and shrinkage force kinetics of high- and low-viscosity dimethacrylateand ormocer-based bulk-fill resin composites. Odontology. 2019;107(1):103-10. http://dx.doi.org/10.1007/s10266-018-0369-y

24. Ehlers V, Gran K, Callaway A, Azrak B, Ernst CP. Oneyear clinical performance of flowable bulk-fill composite vs conventional compomer restorations in primary molars. J Adhes Dent. 2019;21(3):247-54. https://doi.org/10.3290/j.jad.a42519

25. Mosharrafian S, Heidari A, Rahbar P. Microleakage of Two Bulk Fill and One Conventional Composite in Class II Restorations of Primary Posterior Teeth. J Dent (Tehran). 2017;14(3):123-31. Citado em: PMID: 29167683

26. Mosharrafian S, Shafizadeh M, Sharifi Z. Fracture Resistance of a Bulk-Fill and a Conventional Composite and a Combination of Both for Coronal Restoration of Severely Damaged Primary Anterior Teeth. Front Dent. 2019;16(1):69-77. https://dx.doi. org/10.18502\%2Ffid.v16i1.1112

27. Fernandes HGK, Silva R, Marinho MAS, Oliveira POS, Rabelo RJC, Moysés MR. Evolução da resina composta : Revisão da Literatura. Revista da Universidade Vale do Rio Verde [Internet]. 2014;12(2):401-11. Disponível em: http://periodicos.unincor.br/ index.php/revistaunincor/article/view/1465

28. Furness A, Tadros MY, Looney SW, Rueggeberg FA. Effect of bulk/incremental fill on internal gap formation of bulk-fill composites. J Dent. 2014;42(4):439-49. http://dx.doi.org/10.1016/j. jdent.2014.01.005

29. Soares AF, Pinto ACS. Taxa de sucesso de restaurações com resina composta bulk-fill: Revisão de literatura. Id on line Rev Mult Psicol [Internet]. 2019;13(47):397-409. Disponível em: https:// idonline.emnuvens.com.br/id/article/view/2027

30. Chesterman J, Jowett A, Gallacher A, Nixon P. Bulk-fill resinbased composite restorative materials: A review. $\mathrm{Br}$ Dent J. 2017;222(5):337-44. https://doi.org/10.1038/sj.bdj.2017.214

31. Chandrasekhar V, Rudrapati L, Badami V, Tummala M. Incremental techniques in direct composite restoration. J Conserv Dent. 2017;20(6):386-91. https://doi.org/10.4103/jcd.jcd 15716

32. Giachetti L, Russo DS, Bambi C, Grandini R. A review of polymerization shrinkage stress: current techniques for posterior direct resin restorations. J Contemp Dent Pract. 2006;7(4):79-88. Citado em: PMID: 16957794

33. Kim EH, Jung KH, Son SA, Hur B, Kwon YH, Park JK. Effect of resin thickness on the microhardness and optical properties of bulk-fill resin composites. Restor Dent Endod. 2015;40(2):128-35. https://dx.doi.org/10.5395\%2Frde.2015.40.2.128 
34. Veiga BS, Vasconcellos BT. Avaliação do grau de conversão de uma resina convencional e Bulk Fill fotoativadas por distinta fontes de luz. Revista Científica Multidisciplinar Núcleo do Conhecimento [Internet]. 2020;4(3):104-16. Disponível em: https://www.nucleodoconhecimento.com.br/saude/grau-deconversao

35. Holanda LVB, Beserra Neto EP, Costa FMS, Costa EO, Araújo Neto V, Peralta SL. Desempenho das propriedades físicomecânicas das resinas bulk-fill: revisão de literatura. Jornada Odontológica dos Acadêmicos da Católica [Internet]. 2016;2(2). Disponível em: http://publicacoesacademicas.unicatolicaquixada. edu.br/index.php/joac/article/download/998/779

36. Heintze SD, Monreal D, Peschke A. Marginal quality of class II composite restorations placed in bulk compared to an incremental technique: Evaluation with sem and stereomicroscope. J Adhes Dent. 2015;17(2):147-54. https://doi. org/10.3290/j.jad.a33973

37. Van Ende A, De Munck J, Lise DP, Van Meerbeek B. Bulk-Fill Composites: A Review of the Current Literature. J Adhes Dent. 2017;19(2):95-109. https://doi.org/10.3290/j.jad.a38141

38. El-Safty S, Akhtar R, Silikas N, Watts DC. Nanomechanical properties of dental resin-composites. Dent Mater. 2012;28(12):1292-300. http://dx.doi.org/10.1016/j. dental.2012.09.007

39. Clavagio V, Kabbach W. O que pensar da técnica bulk fill? Int J Braz Dent. 2015;11(1):114-23.

40. Kim KH, Ong JL, Okuno O. The effect of filler loading and morphology on the mechanical properties of contemporary composites. J Prosthet Dent. 2002;87(6):642-9. https://doi. org/10.1067/mpr.2002.125179

41. Rosatto CMP, Bicalho AA, Veríssimo C, Bragança GF, Rodrigues $M P$, Tantbirojn D, et al. Mechanical properties, shrinkage stress, cuspal strain and fracture resistance of molars restored with bulk-fill composites and incremental filling technique.

J Dent. 2015;43(12):1519-28. http://dx.doi.org/10.1016/j. jdent.2015.09.007

42. Silva LNC, Silveira CR, Carneiro GKM. Vantagens das resinas bulk fill: revisão da literatura. Revista Saúde Multidisciplinar [Internet]. 2019;5(1):41-7. Disponível em: https://www. fampfaculdade.com.br/wp-content/uploads/2019/04/6VANTAGENS-DAS-RESINAS-BULK-FILL-REVIS\%C3\%830-DALITERATURA.pdf

43. Christensen JJ. Duplicating the form and function of posterior teeth with Class II resin-based composite. Gen Dent. 2012;60(2):104-8. Citado em: PMID: 22414503

44. Boaro LCC, Lopes DP, Souza ASC, Nakano EL, Perez MDA, Pfeifer CS, et al. Clinical performance and chemical-physical properties of bulk fill composites resin - a systematic review and meta-analysis. Dent Mater. 2019;35(10):e249-64. https://doi. org/10.1016/j.dental.2019.07.007
45. Freitas GC, Machado ICP, Oliveira AP, Kasuya AVB, Barata TJE. Resinas compostas: alterações dimensionais em função da composição e do método de irradiação de luz. Robrac [Internet]. 2017;26(77):33-6. Disponível em: https://www.robrac.org.br/seer/ index.php/ROBRAC/article/view/1164

46. Costa MAB, Maior JRS, Guimarães RP, Costa DPTS, Menezes Filho PF, Silva CHV. Restaurações com sistema restaurador Bulk Fill: relato de caso. Rev Gaúch Odontol. 2018;66(4):391-7. https:// doi.org/10.1590/1981-8637201800040000143505

47. Barutcigil Ç, Barutcigil K, Özarslan MM, Dündar A, Yilmaz B. Color of bulk-fill composite resin restorative materials. J Esthet Restor Dent. 2018;30(2):E3-8. https://doi.org/10.1111/jerd.12340

48. Almeida L, Santin DC, Maran BM, Naufel FS, Schmitt VL. Avaliação do manchamento e da rugosidade superficial de materiais restauradores diretos após diferentes sistemas de polimento: estudo in vitro. Rev Odontol UNESP. 2019;48:e20180096. https://doi.org/10.1590/1807-2577.09618

49. Miletic V, Marjanovic J, Veljovic DN, Stasic JN, Petrovic V. Color stability of bulk-fill and universal composite restorations with dissimilar dentin replacement materials. J Esthet Restor Dent. 2019;31(5):520-8. https://doi.org/10.1111/jerd.12529

50. Veloso SRM, Lemos CAA, Moraes SLD, Vasconcelos BCE, Pellizzer EP, Monteiro GQM. Clinical performance of bulk-fill and conventional resin composite restorations in posterior teeth: a systematic review and meta-analysis. Clin Oral Investig. 2019;23(1):221-33. https://doi.org/10.1007/s00784-018-2429-7

51. Abdulmajeed AA, Donovan TE, Cook R, Sulaiman TA. Effect of Preheating and Fatiguing on Mechanical Properties of Bulk-fill and Conventional Composite Resin. Oper Dent. 2020;45(4):387-95. https://doi.org/10.2341/19-092-I

52. Sunbul HA, Silikas N, Watts DC. Surface and bulk properties of dental resin- composites after solvent storage. Dent Mater. 2016;32(8):987-97. http://dx.doi.org/10.1016/j.dental.2016.05.007

53. Rauber GB, Bernardon JK, Vieira LCC, Maia HP, Horn F, Roesler CRM. In vitro fatigue resistance of teeth restored with bulk fill versus conventional composite resin. Braz Dent J. 2016;27(4):4527. https://doi.org/10.1590/0103-6440201600836

54. Papadogiannis D, Tolidis K, Gerasimou P, Lakes R, Papadogiannis $Y$. Viscoelastic properties, creep behavior and degree of conversion of bulk fill composite resins. Dent Mater. 2015;31(12):1533-41. http://dx.doi.org/10.1016/j. dental.2015.09.022 